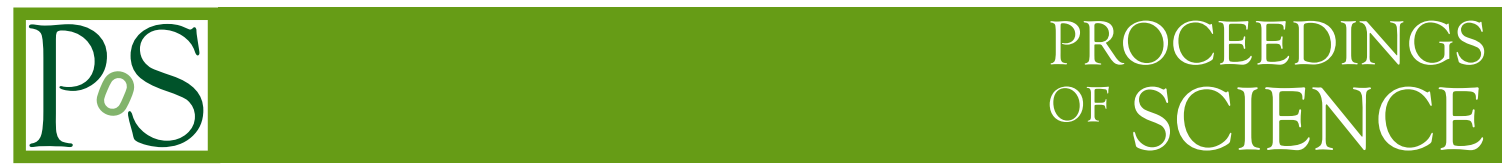

\title{
Yield of heavy hyperons and antihyperons in DIS
}

\author{
Natalia Rossiyskaya*, on behalf of the COMPASS collaboration \\ Joint Institute for Nuclear Research \\ E-mail: Natalia.Rossiyskaya@cern.ch
}

\begin{abstract}
The yields of heavy hyperons and antihyperons have been studied in deep inelastic scattering at the COMPASS experiment at CERN. The data were collected in 2003-2004 using a $160 \mathrm{GeV}$ polarised muon beam scattered off a large polarised ${ }^{6} \mathrm{LiD}$ target. Signals from $\Sigma^{ \pm}(1385)$ and $\bar{\Sigma}^{ \pm}(1385)$ decays were reconstructed. The ratios of $\Sigma^{ \pm} / \Lambda, \bar{\Sigma}^{ \pm} / \bar{\Lambda}$ and $\Lambda / \bar{\Lambda}$ were determined. These ratios were used to tune parameters of the LEPTO generator. A comparison with the yields of the heavy hyperons in the low $Q^{2}$ region was done.
\end{abstract}

XVIII International Workshop on Deep-Inelastic Scattering and Related Subjects April 19 -23, 2010

Convitto della Calza, Firenze, Italy

\footnotetext{
* Speaker.
} 


\section{Introduction}

It is important to measure the yields of heavy hyperons and antihyperons in deep inelastic scattering (DIS) for understanding the dynamics of hyperon production and polarization. The decays of heavy hyperons are also important for $\Lambda$ and $\bar{\Lambda}$ production and polarization. It is known [1] that a significant part of the $\Lambda$ hyperons in DIS is produced indirectly, via decays of heavy hyperons such as $\Sigma^{0}, \Sigma(1385), \Xi$ etc. The polarization of these indirect $\Lambda$ hyperons may influence the $\Lambda$ polarization. On the other hand, the role of the indirect $\bar{\Lambda}$ hyperons, forming in the decays of heavy antihyperons, is not known. No measurements of the heavy antihyperon yields in the DIS exist before.

The yields of the heavy hyperons in the neutrino DIS were measured by the NOMAD collaboration [2]. They have found strong deviation of the measured yields from the Monte Carlo predictions. The experimental yields were used to tune the parameters of the LEPTO generator.

\section{The experimental setup}

COMPASS is a fixed-target experiment at the CERN Super Proton Synchrotron (SPS) using high-energy muon and hadron beams. The apparatus is described in detail in [3]. The data used in the present analysis were collected during the years 2003-2004 with $160 \mathrm{GeV}$ polarised $\mu^{+}$ scattering off a polarised ${ }^{6} \mathrm{LiD}$ target. The polarized target of the COMPASS spectrometer consists of two cells with a length of $60 \mathrm{~cm}$ each and a diameter of $3 \mathrm{~cm}$. They are placed along the beam direction with a distance of $10 \mathrm{~cm}$ between them. Both cells are placed inside a conductive solenoid with magnetic field $2.5 \mathrm{~T}$ and temperature about $50 \mathrm{mK}$. The data from both longitudinal target spin orientations were recorded simultaneously and averaged in the present analysis.

The beam tracks were reconstructed in a beam telescope consisting of silicon microstrip and scintillating fibre detectors. The interaction products are observed in a forward magnetic spectrometer with two stages for low and high momenta, respectively, each equipped with high-resolution tracking detectors and electromagnetic and hadron calorimetry. Information from the RICH detector installed in the first stage of the spectrometer was not used in this analysis. Muons were identified downstream of iron and concrete walls.

\section{The data analysis}

The event selection requires a reconstructed interaction vertex defined by the incoming and the scattered muon located inside the target. DIS events are selected by cuts on the photon virtuality $\left(Q^{2}>1(\mathrm{GeV} / \mathrm{c})^{2}\right)$ and on the fractional energy of the virtual photon $(0.2<y<0.9)$. The data sample consists of $8.67 \cdot 10^{7}$ DIS events from the 2003 run and $22.5 \cdot 10^{7}$ DIS events from the 2004 run.

We studied following decays of heavy hyperons and antihyperons:

$$
\begin{array}{ll}
\mu^{+}+d \rightarrow \mu^{+}+\Sigma^{ \pm}(1385)+X & \left(\Sigma^{ \pm}(1385) \rightarrow \Lambda+\pi^{ \pm}\right) \\
\mu^{+}+d \rightarrow \mu^{+}+\bar{\Sigma}^{ \pm}(1385)+X & \left(\bar{\Sigma}^{ \pm}(1385) \rightarrow \bar{\Lambda}+\pi^{ \pm}\right)
\end{array}
$$


In these processes the heavy hyperon decays due to the strong interaction in the primary vertex, whereas the secondary vertex is a signature for the $\Lambda(\bar{\Lambda})$ weak decays in to $p \pi^{-}\left(\bar{p} \pi^{+}\right)$.

The analysis is starts from the reconstruction of the vector of the momentum of the $\Lambda(\bar{\Lambda})$ hyperon. The $\Lambda$ and $\bar{\Lambda}$ events were selected by requiring two hadron tracks forming a secondary vertex in addition to the incoming and scattered muon at the primary vertex. The criteria for the $\Lambda(\bar{\Lambda})$ selection were basically the same as in the analysis of the longitudinal spin transfer to $\Lambda(\bar{\Lambda})$ [4]. We select $\Lambda(\bar{\Lambda})$ events in the current fragmentation region $0.05<x_{F}<1.0$, where $x_{F}$ is the Feynman variable $x_{F}=2 p_{L} / W, p_{L}$ is the particle longitudinal momentum in the hadronic centre-of-mass system, whose invariant mass is $W$. The angle $\theta_{\text {coll }}$ between the hyperon momentum and the line connecting the primary and the secondary vertex is required to be $\theta_{\text {coll }}<0.01 \mathrm{rad}$. This cut selects events with the correct direction of the hyperon momentum vector with respect to the primary vertex resulting in a reduction of the combinatorial background. A cut on the transverse momentum $p_{t}$ of the decay products with respect to the hyperon direction of $p_{t}>23 \mathrm{MeV} / \mathrm{c}$ is applied in order to reject $e^{+} e^{-}$pairs due to $\gamma$ conversion.

The resulting $p \pi^{-}$and $\bar{p} \pi^{+}$invariant mass spectra are presented in Fig. 1. They were fitted within $1.095-1.140 \mathrm{GeV}$ interval by the sum a Gaussian for the $\Lambda$ signal and the third order polynomial for the background. The total number of events after all selection cuts are $N(\Lambda)=99667 \pm 385$ and $N(\bar{\Lambda})=60056 \pm 322$. This is much more then in all previous experiments on $\Lambda$ and $\bar{\Lambda}$ production in DIS $[1,2,5]$.
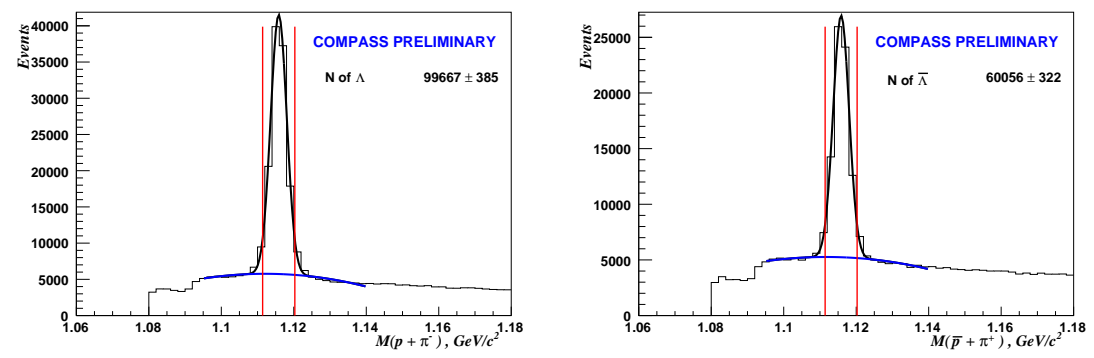

Figure 1: Invariant mass distributions of $p \pi^{-}$and $\bar{p} \pi^{+}$. The vertical lines show the $(2 \sigma)$ region selected for further analysis.

The $\Sigma^{ \pm}(1385)$ and $\bar{\Sigma}^{ \pm}(1385)$ resonances decay in $\Lambda \pi^{+}$and $\bar{\Lambda} \pi^{-}$in the primary vertex. The invariant mass distributions of $\Lambda \pi^{+}$and $\bar{\Lambda} \pi^{-}$for experimental data are shown in Fig. 2. The distributions have been fitted by a sum of the Breit-Wigner convoluted with the gaussian for the signal

$$
R(x)=\frac{\Gamma}{(2 \cdot \pi)^{3 / 2}} \cdot \int \frac{N d t}{(t-M)^{2}+\left(\frac{\Gamma}{2}\right)^{2}} \cdot e^{-\frac{1}{2}\left(\frac{t-x}{\sigma}\right)^{2}}
$$

and the background parametrised as

$$
B(x)=a \cdot\left(x-M_{t h}\right)^{b} \cdot e^{-c \cdot\left(x-M_{t h}\right)^{d}} .
$$

Here a,b,c,d are the fit parameters, the mass $M$ and the width $\Gamma$ of the hyperon were fixed according to the Particle Data Group Tables and $M_{t h}=1254 \mathrm{MeV}$ is the sum of the $\Lambda$ and $\pi$ mass. 

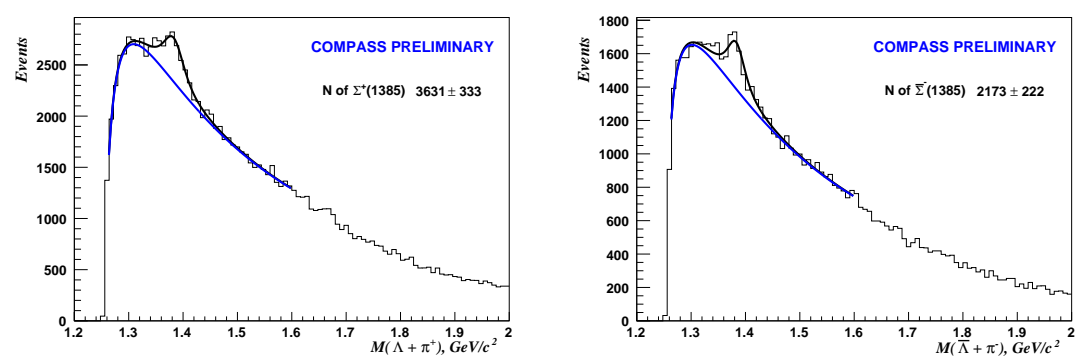

Figure 2: Invariant mass distributions of $\Lambda \pi^{+}$and $\bar{\Lambda} \pi^{-}$.

The invariant mass distributions of $\Lambda \pi^{-}$and $\bar{\Lambda} \pi^{+}$for experimental data are shown in Fig. 3. One can see two peaks: the narrow one is due to part of the decays of $\Xi^{-}\left(\bar{\Xi}^{+}\right)$, which passed the collinearity cut. The collinearity cut selects events with the $\Lambda(\bar{\Lambda})$ momentum directed to the primary vertex. Effectively this cut selects strong decays of the heavy hyperons. However, some part of the weak hyperon decays, such as $\Xi^{-}(1321) \rightarrow \Lambda+\pi^{-}\left(\bar{\Xi}^{+}(1321) \rightarrow \bar{\Lambda}+\pi^{+}\right)$is leaking through the collinearity cut. The correct determination of the weak hyperon decays needs a special investigation. At the present work we refrain from the determination of the $\Xi$ yields.

The wider peak at Fig. 3 is from $\Sigma^{-}\left(\bar{\Sigma}^{+}\right)$. The $\Xi$ peak was fitted by a gaussian, the $\Sigma$ peak was fitted by convolution of Breit-Wigner and gaussian (3.1).
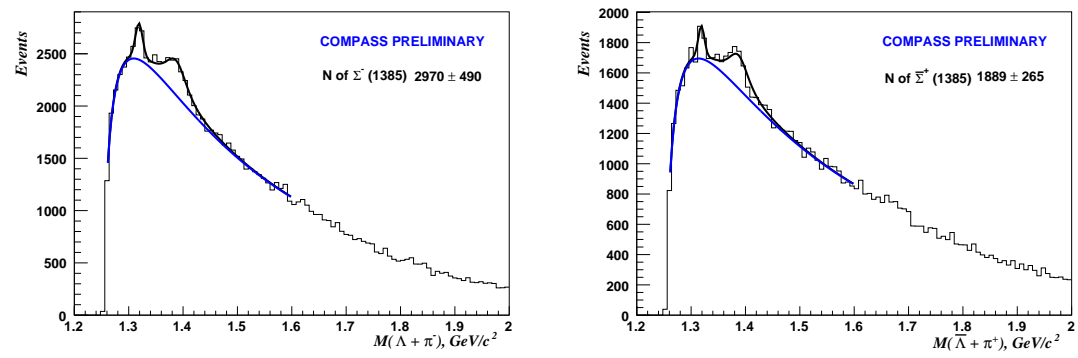

Figure 3: Invariant mass distributions of $\Lambda \pi^{-}$and $\bar{\Lambda} \pi^{+}$.

The total number of events are $N\left(\Sigma^{+}\right)=3631 \pm 311, N\left(\Sigma^{-}\right)=2970 \pm 490, N\left(\bar{\Sigma}^{-}\right)=2173 \pm$ 222 and $N\left(\bar{\Sigma}^{+}\right)=1889 \pm 265$.

For a study of the systematic effects we evaluate the background using a mixed event method, in which the shape of the background distribution in the $\Lambda \pi$ invariant mass is determined combining $\Lambda$ and $\pi$ from different events of the same topology. The systematic error connected with the particular choice of the selection cut of the $\Lambda(\bar{\Lambda})$ sample is estimated by changing the interval from the mean value of the $\Lambda(\bar{\Lambda})$ peak from $\pm 2 \sigma$ to $\pm 2.5 \sigma$ and $\pm 1.5 \sigma$. In the result, the systematic error turns out to be less or comparable with the statistical error.

The ratios for the yields of heavy hyperons and antihyperons to $\Lambda$ and $\bar{\Lambda}$ are the following:

$$
\begin{aligned}
& R^{+}=\Sigma^{+}(1385) / \Lambda=0.055 \pm 0.005(\text { stat }) \pm 0.0045(\text { syst }) \\
& \bar{R}^{-}=\bar{\Sigma}^{-}(1385) / \bar{\Lambda}=0.047 \pm 0.006(\text { stat }) \pm 0.0053(\text { syst }) \\
& R^{-}=\Sigma^{-}(1385) / \Lambda=0.056 \pm 0.009(\text { stat }) \pm 0.0074(\text { syst }) \\
& \bar{R}^{+}=\bar{\Sigma}^{+}(1385) / \bar{\Lambda}=0.039 \pm 0.006(\text { stat }) \pm 0.0064(\text { syst })
\end{aligned}
$$


These ratios are corrected for the experimental acceptance evaluated by a Monte Carlo simulation. To evaluate the acceptance correction the yields of hyperons after reconstruction have been compared with those from the LEPTO generator. The acceptance correction includes the correction for all hyperon and $\Lambda$ decays. The main conclusion from the data (3.3)-(3.6) is that the percentage of the indirect $\Lambda$ from the decays of $\Sigma(1385)$ in the total $\Lambda$ sample is similar to the percentage of indirect $\bar{\Lambda}$ from decays of $\bar{\Sigma}(1385)$. However, the percentage of indirect $\bar{\Lambda}$ from $\bar{\Sigma}(1385)$ decays is slightly lower. It is important to note, that we compare the yields for $\Lambda$ and $\bar{\Lambda}$ hyperons created in the current fragmentation region $\left(x_{F}>0.05\right)$.

The yields of the heavy hyperons in the neutrino DIS were measured by the NOMAD collaboration [2]. The ratio $\Sigma^{+}(1385) / \Lambda$ was found to be $R^{+}=0.058 \pm 0.011$, in agreement with (3.3). The ratio $\Sigma^{-}(1385) / \Lambda$ was $R^{-}=0.026 \pm 0.008$, two times smaller than (3.5). The difference between $R^{+}$and $R^{-}$is natural for the neutrino-nucleon interaction. No measurements of the heavy antihyperon yields in the DIS exist before.

It is interesting how the yields of heavy hyperons change if one refrains from DIS cuts. We remove the demands that the $Q^{2}>1(\mathrm{GeV} / c)^{2}$ and $0.2<y<0.9$. The data sample increases by a factor 10. In total, there are $N(\Lambda)=1208413 \pm 1312$ and $N(\bar{\Lambda})=654387 \pm 1067$. However, no change in the ratios $\Sigma / \Lambda$ and $\bar{\Sigma} / \bar{\Lambda}$ is found within the statistical errors. In Table 1 the ratios $\Sigma / \Lambda$ and $\bar{\Sigma} / \bar{\Lambda}$ with and without the DIS cuts are compared.

Table 1: The ratios of the hyperon yields for events with and without the DIS cuts

\begin{tabular}{|l|l|}
\hline$\Sigma / \Lambda$ (no cut) $/ \Sigma / \Lambda$ (DIS cut) & \\
\hline$\Sigma^{+} / \Lambda$ & $1.03 \pm 0.08$ \\
\hline $\bar{\Sigma}^{-} / \bar{\Lambda}$ & $0.97 \pm 0.11$ \\
\hline$\Sigma^{-} / \Lambda$ & $1.03 \pm 0.16$ \\
\hline $\bar{\Sigma}^{+} / \bar{\Lambda}$ & $0.97 \pm 0.13$ \\
\hline
\end{tabular}

The experimental relative yields (3.3)-(3.6) were used to tune the parameters of the LEPTO generator. The COMPASS Monte Carlo code is based on the LEPTO 6.5.1 generator [6] providing DIS events which are passed through a GEANT-based apparatus simulation programme and the same chain of reconstruction procedures as the experimental events. In Table 2 a comparison between the default LEPTO parameters, the NOMAD and the COMPASS ones is given. The results of the tuning on the COMPASS data are given in the last column.

The tuned LEPTO correctly reproduce the experimentally measured yields of $\Sigma(1385)$ and $\bar{\Sigma}(1385)$ as well as the ratios $\Lambda / \bar{\Lambda}$ and $K / \Lambda$. The new LEPTO parameters strongly influence on the theoretical predictions of the $\Lambda(\bar{\Lambda})$ polarization. To demonstrate the effect of the new LEPTO parameters the spin transfer to $\Lambda$ and $\bar{\Lambda}$ is calculated using the theoretical model of [7]. In Fig. 4 the $x_{F}$ dependence of the spin transfer to the $\Lambda$ and $\bar{\Lambda}$ calculated for the new (left) and old (right) LEPTO parameters is shown. One could see that the calculation with the tuned LEPTO predicts different $x_{F}$ dependence of the spin transfer than the default LEPTO. The calculations with the tuned LEPTO shows no dependence of the $\Lambda$ spin transfer with the $x_{F}$. The Fig. 4 demonstrates that the knowledge of the heavy hyperons yields is important for the correct interpretation of the $\Lambda$ and $\bar{\Lambda}$ hyperons polarization. 
Table 2: Comparison between the default LEPTO parameters[6], NOMAD [2] and present tuning.

\begin{tabular}{llll}
\hline Parameters & Default & NOMAD & COMPASS \\
\hline PARJ(1) & 0.1 & 0.05 & 0.055 \\
PARJ(2) & 0.3 & 0.21 & 0.2 \\
PARJ(3) & 0.4 & 0.07 & 0.97 \\
PARJ(4) & 0.05 & 0.001 & 0.007 \\
PARJ(5) & 0.5 & 0.97 & 3.0 \\
PARJ(6) & 0.5 & 0.5 & 0.5 \\
PARJ(7) & 0.5 & 0.39 & 0.97 \\
\hline
\end{tabular}
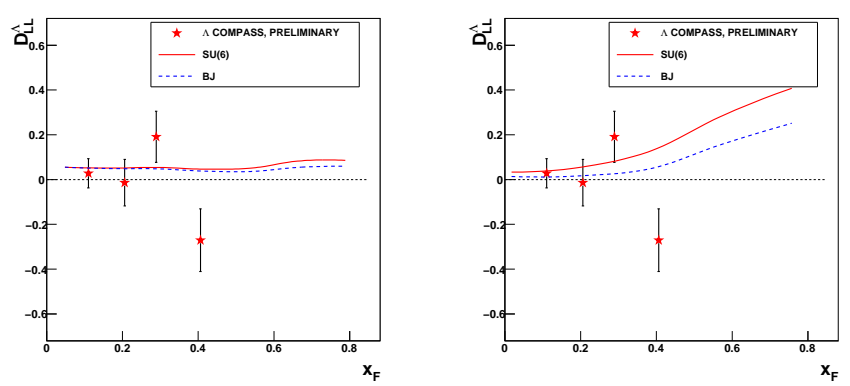

Figure 4: Comparison of the spin transfer to the $\Lambda$ calculated for the new (left) and old (right) LEPTO parameters. The red lines corresponds to the SU(6) model for the $\Lambda$ spin structure, whereas the blue lines corresponds to the calculations [7] in the BJ-model [8].The experimental data are from [4].

In conclusion, the yields of the $\Sigma(1385)$ and $\bar{\Sigma}(1385)$ in DIS are measured. The percentage of the indirect $\Lambda$ from the decays of $\Sigma(1385)$ in the total $\Lambda$ sample is similar as percentage of indirect $\bar{\Lambda}$ from decays of $\bar{\Sigma}(1385)$. The yields are used to tune the LEPTO generator parameters. Theoretical calculations with the tuned LEPTO parameters demonstrate substantial impact on the $\Lambda$ and $\bar{\Lambda}$ spin transfer. Removing DIS cuts does not change the ratios $\Sigma / \Lambda$ and $\bar{\Sigma} / \bar{\Lambda}$ within the statistical errors.

\section{References}

[1] HERMES Collaboration, A. Airapetyan et al., Phys. Rev. D74 072004 (2006).

[2] NOMAD Collaboration, P. Astier et al., Nucl.Phys. B621 (2002) 3.

[3] COMPASS Collaboration, P.Abbon et al., Nucl. Instrum. Meth. A577 (2007) 455.

[4] COMPASS Collaboration, M.Alekseev et al., Eur.Phys.J. C64 (2009) 171.

[5] E665 Collaboration, M.R.Adams et al., Eur. Phys. J. C17 (2000) 263.

[6] A.E.G.Ingelman, and J.Rathsman, Comp. Phys. Commun. 101, 10.

[7] J.Ellis, A.M.Kotzinian, D.Naumov, M.G.Sapozhnikov, Eur.Phys.J. C52, 283 (2007).

[8] M. Burkardt, R. L. Jaffe, Phys. Rev. Lett. 702537 (1993). 